\title{
Multi-objective optimization for delivering perishable products with mixed time windows
}

\author{
Wang, X.P. ${ }^{a, b,{ }^{*}}$, Wang, M. ${ }^{b}$, Ruan, J.H. ${ }^{c}$, Li, Y. ${ }^{a}$ \\ anstitute of Systems Engineering, Dalian University of Technology, Dalian, Liaoning, P.R. China \\ ${ }^{b}$ School of Business, Dalian University of Technology, Panjin, Liaoning, P.R. China \\ ${ }^{c}$ College of Economics and Management, Northwest A\&F University, Yangling, Shanxi, P.R. China
}

\begin{abstract}
A B S T R A C T
Perishable products generally have a short shelf life, and the freshness often depends on the postharvest time. The freshness of perishable products can ensure better customer satisfaction. Owing to the deterioration of perishable goods, the complexity of the corresponding vehicle routing problem (VRP) increases, because time delay will lead to serious costs. In this study, we are concerned with not only time-sensitive spoilage rates with mixed time windows, but also the delay costs in delivering perishable products. This study proposes a multi-objective VRP optimization model with mixed time windows and perishability (MO-VRPMTW-P) to minimize the distribution costs and maximize the freshness of perishable products. Then, in view of the fresh products orders space and time characteristics, we propose a heuristic algorithm (ST-VNSGA) composed of a variable neighbourhood search (VNS) method and a genetic algorithm (GA) considering the spatio-temporal (ST) distance to solve the complex multi-objective problem. The solution algorithms are evaluated through a series of experiments. We illustrate the performance and efficiency comparisons of ST-VNSGA with the method without spatio-temporal strategy algorithm and NSGA-II algorithm. It is demonstrated that the proposed ST-VNSGA algorithm can lead to a substantial decrease in the computation time and major improvements in solutions quality, thus revealing the efficiency of considering the spatio-temporal strategy with mixed time windows.
\end{abstract}

\author{
ARTICLE INFO \\ Keywords: \\ Perishable products distribution; \\ Multi-objective optimization; \\ Mixed time windows; \\ Freshness; \\ Heuristic algorithm; \\ Spatio-temporal distance \\ *Corresponding author: \\ wxp@dlut.edu.cn \\ (Wang, X.P.) \\ Article history: \\ Received 13 December 2017 \\ Revised 20 August 2018 \\ Accepted 24 August 2018
}

\section{References}

[1] Hwang, H.-S. (1999). A food distribution model for famine relief, Computers \& Industrial Engineering, Vol. 37, No. 1-2, 335-338, doi: 10.1016/S0360-8352(99)00087-X.

[2] Teunter, R.H., Flapper, S.D.P. (2003). Lot-sizing for a single-stage single-product production system with rework of perishable production defectives, OR Spectrum, Vol. 25, No. 1, 85-96, doi: 10.1007/s00291-002-0105-3.

[3] Amorim, P., Almada-Lobo, B. (2014). The impact of food perishability issues in the vehicle routing problem, Computers \& Industrial Engineering, Vol. 67, 223-233, doi: 10.1016/i.cie.2013.11.006.

[4] Song, B.D., Ko, Y.D. (2016). A vehicle routing problem of both refrigerated- and general-type vehicles for perishable food products delivery, Journal of Food Engineering, Vol. 169, 61-71, doi: 10.1016/i.jfoodeng.2015.08.027.

[5] Hu, H., Zhang, Y., Zhen, L. (2017). A two-stage decomposition method on fresh product distribution problem, International Journal of Production Research, Vol. 55, No. 16, 4729-4752, doi: 10.1080/00207543.2017.1292062.

[6] Tarantilis, C.D., Kiranoudis, C.T. (2001). A meta-heuristic algorithm for the efficient distribution of perishable foods, Journal of Food Engineering, Vol. 50, No. 1, 1-9, doi: 10.1016/S0260-8774(00)00187-4.

[7] Tarantilis, C.D., Kiranoudis, C.T. (2002). Distribution of fresh meat, Journal of Food Engineering, Vol. 51, No. 1, 8591, doi: 10.1016/S0260-8774(01)00040-1. 
[8] Zhang, G., Habenicht, W., Spie $\beta$, W.E.L. (2003). Improving the structure of deep frozen and chilled food chain with tabu search procedure, Journal of Food Engineering, Vol. 60, No. 1, 67-79, doi: 10.1016/S0260-8774(03) 00019-0.

[9] Faulin, J. (2003). Applying MIXALG procedure in a routing problem to optimize food product delivery, Omega, Vol. 31, No. 5, 387-395, doi: org/10.1016/S0305-0483(03)00079-3.

[10] Chen, H.-K., Hsueh, C.-F., Chang, M.-S. (2009). Production scheduling and vehicle routing with time windows for perishable food products, Computers \& Operations Research, Vol. 36, No. 7, 2311-2319, doi: 10.1016/j.cor.2008. 09.010 .

[11] Osvald, A., Zadnik Stirn, L. (2008). A vehicle routing algorithm for the distribution of fresh vegetables and similar perishable food, Journal of Food Engineering, Vol. 85, No. 2, 285-295, doi: 10.1016/i.jfoodeng.2007.07.008.

[12] Hsu, C.-I., Hung, S.-F., Li, H.-C. (2007). Vehicle routing problem with time-windows for perishable food delivery, Journal of Food Engineering, Vol. 80, No. 2, 465-475, doi: 10.1016/j.jfoodeng.2006.05.029.

[13] Ruan, J., Shi, Y. (2016). Monitoring and assessing fruit freshness in IOT-based e-commerce delivery using scenario analysis and interval number approaches, Information Sciences, Vol. 373, 557-570, doi: 10.1016/j.ins.2016. $\underline{07.014}$

[14] Rong, A., Akkerman, R., Grunow, M. (2011). An optimization approach for managing fresh food quality throughout the supply chain, International Journal of Production Economics, Vol. 131, No. 1, 421-429, doi: 10.1016/j.ijpe. 2009.11.026.

[15] Amorim, P., Günther, H.-O., Almada-Lobo, B. (2012). Multi-objective integrated production and distribution planning of perishable products, International Journal of Production Economics, Vol. 138, No. 1, 89-101, doi: 10.1016/j.ijpe.2012.03.005.

[16] Prindezis, N., Kiranoudis, C.T., Marinos-Kouris, D. (2003). A business-to-business fleet management service provider for central food market enterprises, Journal of Food Engineering, Vol. 60, No. 2, 203-210, doi: 10.1016/ S0260-8774(03)00041-4.

[17] Hasani, A., Zegordi, S.H., Nikbakhsh, E. (2012). Robust closed-loop supply chain network design for perishable goods in agile manufacturing under uncertainty, International Journal of Production Research, Vol. 50, No. 16, 4649-4669, doi: 10.1080/00207543.2011.625051.

[18] Govindan, K., Jafarian, A., Khodaverdi, R., Devika, K. (2014). Two-echelon multiple-vehicle location-routing problem with time windows for optimization of sustainable supply chain network of perishable food, International Journal of Production Economics, Vol. 152, 9-28, doi: 10.1016/i.ijpe.2013.12.028.

[19] Claassen, G.D.H., Gerdessen, J.C., Hendrix, E.M.T., van der Vorst, J.G.A.J. (2016). On production planning and scheduling in food processing industry: Modelling non-triangular setups and product decay, Computers \& Operations Research, Vol. 76, 147-154, doi: 10.1016/i.cor.2016.06.017.

[20] Pahl, J., Voß, S. (2014). Integrating deterioration and lifetime constraints in production and supply chain planning: A survey, European Journal of Operational Research, Vol. 238, No. 3, 654-674, doi: 10.1016/i.ejor.2014. $\underline{01.060 .}$.

[21] de Keizer, M., Akkerman, R., Grunow, M., Bloemhof, J.M., Haijema, R., van der Vorst, J.G.A.J. (2017). Logistics network design for perishable products with heterogeneous quality decay, European Journal of Operational Research, Vol. 262, No. 2, 535-549, doi: 10.1016/j.ejor.2017.03.049.

[22] Albrecht, W., Steinrücke, M. (2018). Coordinating continuous-time distribution and sales planning of perishable goods with quality grades, International Journal of Production Research, Vol. 56, No. 7, 2646-2665, doi: 10.1080/ 00207543.2017 .1384584

[23] Devapriya, P., Ferrell, W., Geismar, N. (2017). Integrated production and distribution scheduling with a perishable product, European Journal of Operational Research, Vol. 259, No. 3, 906-916, doi: 10.1016/i.ejor.2016.09.019.

[24] Moon, I., Lee, S. (2000). The effects of inflation and time-value of money on an economic order quantity model with a random product life cycle, European Journal of Operational Research, Vol. 125, No. 3, 588-601, doi: 10.1016/S0377-2217(99)00270-2.

[25] Chen, J.-M., Lin, C.-S. (2002). An optimal replenishment model for inventory items with normally distributed deterioration, Production Planning \& Control, Vol. 13, No. 5, 470-480, doi: 10.1080/09537280210144446.

[26] Qi, M., Lin, W.-H., Li, N., Miao, L. (2012). A spatiotemporal partitioning approach for large-scale vehicle routing problems with time windows, Transportation Research Part E: Logistics and Transportation Review, Vol. 48, No. 1, 248-257, doi: 10.1016/i.tre.2011.07.001. 
APEM
Advances in Production Engineering \& Management Letnik 13 | Številka 3 | September 2018 | Strani 321-332 https://doi.org/10.14743/apem2018.3.293
ISSN 1854-6250

Spletna stran: apem-journal.org Izvirni znanstveni članek

\title{
Večkriterijska optimizacija za dostavo pokvarljivih izdelkov z mešanimi časovnimi okni
}

\author{
Wang, X.P. ${ }^{\text {a,b, }}{ }^{*}$, Wang, M. ${ }^{b}$, Ruan, J.H. ${ }^{c}$, Li, Y. ${ }^{a}$ \\ ${ }^{a}$ Institute of Systems Engineering, Dalian University of Technology, Dalian, Liaoning, P.R. China \\ ${ }^{b}$ School of Business, Dalian University of Technology, Panjin, Liaoning, P.R. China \\ ${ }^{c}$ College of Economics and Management, Northwest A\&F University, Yangling, Shanxi, P.R. China
}

\section{POVZETEK}

Pokvarljivi izdelki imajo na splošno kratek rok trajanja, njihova svežina pa je pogosto odvisna od časovnega obdobja od žetve. Svežina pokvarljivih izdelkov lahko zagotovi večje zadovoljstvo strank. Z upoštevanjem poslabšanja kakovosti pokvarljivega blaga se poveča kompleksnost problema usmerjanja dostavnega vozila (VRP), saj časovna zamuda povzroči resne stroške. Študija se ne ukvarja le s časovno občutljivimi stopnjami kvarjenja izdelkov z mešanimi časovnimi okni, temveč tudi s stroškom zamude pri dobavi pokvarljivih izdelkov. Študija predlaga večkriterijski optimizacijski model z mešanimi časovnimi okni in pokvarljivostjo (MO-VRPMTW-P) za zmanjšanje stroškov distribucije in zvečanje svežine pokvarljivih izdelkov. Glede na prostorsko razporeditev naročil svežih izdelkov in časovne značilnosti predlagamo hevristični algoritem (ST-VNSGA), sestavljen iz algoritma za preiskovanje s spremenljivo soseščino (VNS), genetskega algoritma (GA) in prostorsko-časovne (ST) razdalje za reševanje večkriterijskega optimizacijskega problema. Primerjani algoritmi so bili ovrednoteni s poskusi. V raziskavi je prikazana uspešnost in učinkovitost ST-VNSGA v primerjavi z algoritmom brez prostorsko-časovne strategije in algoritmom NSGA-II. Izkazalo se je, da lahko predlagani algoritem STVNSGA znatno zmanjša potreben računski čas in bistveno izboljša kakovost rešitev, kar opravičuje upoštevanje prostorsko-časovne strategije pri reševanju problemov z mešanimi časovnimi okni.

\section{PODATKI O ČLANKU}

Ključne besede:

Distribucija pokvarljivih izdelkov;

Večkriterijske optimizacije;

Mešana časovna okna;

Svežina;

Hevristični algoritmi;

Prostorsko-časovna razdalja

*Kontaktna oseba: wxp@dlut.edu.cn (Wang, X.P.)

Zgodovina članka:

Prejet 13. decembra 2017

Popravljen 20. avgusta 2018

Sprejet 24. avgusta 2018 